\title{
Ccreative
commons
}

ISSN 1855-3966 (printed edn.), ISSN 1855-3974 (electronic edn.)

\author{
ARS MATHEMATICA CONTEMPORANEA 15 (2018) 113-126 \\ https://doi.org/10.26493/1855-3974.1414.58b \\ (Also available at http://amc-journal.eu)
}

\section{Coloring properties of categorical product of general Kneser hypergraphs*}

\author{
Roya Abyazi Sani, Meysam Alishahi ${ }^{\dagger}$ \\ School of Mathematical Sciences, Shahrood University of Technology, \\ Shahrood, Iran \\ Ali Taherkhani \\ Department of Mathematics, Institute for Advanced Studies in Basic Sciences (IASBS), \\ Zanjan 45137-66731, Iran
}

Received 26 May 2017, accepted 9 October 2017, published online 6 March 2018

\begin{abstract}
More than 50 years ago Hedetniemi conjectured that the chromatic number of categorical product of two graphs is equal to the minimum of their chromatic numbers. This conjecture has received a considerable attention in recent years. Hedetniemi's conjecture was generalized to hypergraphs by Zhu in 1992. Hajiabolhassan and Meunier, in 2016, introduced the first nontrivial lower bound for the chromatic number of categorical product of general Kneser hypergraphs and using this lower bound, they verified Zhu's conjecture for some families of hypergraphs. In this paper, we shall present some colorful type results for the coloring of categorical product of general Kneser hypergraphs, which generalize the Hajiabolhassan-Meunier result. Also, we present a new lower bound for the chromatic number of categorical product of general Kneser hypergraphs which can be much better than the Hajiabolhassan-Meunier lower bound. Using this lower bound, we enrich the family of hypergraphs satisfying Zhu's conjecture.
\end{abstract}

Keywords: Categorical product, chromatic number, Hedetniemi's conjecture, general Kneser hypergraph.

Math. Subj. Class.: 05C15

\footnotetext{
*We thank the two anonymous referees for the carefully reading our paper and for all their useful remarks that helped in improving the presentation of the paper.

${ }^{\dagger}$ Corresponding author.

E-mail address: roya.abyazisani@shahroodut.ac.ir (Roya Abyazi Sani), meysam_alishahi@shahroodut.ac.ir (Meysam Alishahi), ali.taherkhani@iasbs.ac.ir (Ali Taherkhani)
}

(a) (i) This work is licensed under http://creativecommons.org/licenses/by/3.0/ 


\section{Introduction and main results}

For two graphs $G$ and $H$, their categorical product $G \times H$ is the graph defined on the vertex set $V(G) \times V(H)$ such that two vertices $(g, h)$ and $\left(g^{\prime}, h^{\prime}\right)$ are adjacent whenever $g g^{\prime} \in$ $E(G)$ and $h h^{\prime} \in E(H)$. The categorical product is the product involved in the famous long-standing conjecture posed by Hedetniemi which states that the chromatic number of $G \times H$ is equal to the minimum of $\chi(G)$ and $\chi(H)$. It was shown that the conjecture is true for several families of graphs, but it is wide open in general (see Tardif [21] and Zhu [23]). In spite of being investigated in several articles, there is no substantial progress in solving this conjecture. This conjecture was generalized to the case of hypergraphs by Zhu [22].

A hypergraph $\mathcal{H}$ is an ordered pair $(V(\mathcal{H}), E(\mathcal{H}))$ where $V(\mathcal{H})$ is a set of vertices, and $E(\mathcal{H})$ is a family of nonempty subsets of $V(\mathcal{H})$. The elements of $E(\mathcal{H})$ are called edges. All hypergraphs considered in the paper have no multiple edges and $E(\mathcal{H})$ is thus a usual set. For a subset $S \subseteq V(\mathcal{H})$, the subhypergraph induced by $S$, denoted by $\mathcal{H}[S]$, is a hypergraph with vertex set $S$ and edge set $\{e \in E(\mathcal{H}): e \subseteq S\}$. A hypergraph $\mathcal{H}$ is said to be $r$-uniform if $E(\mathcal{H})$ is a family of $r$-subsets of $V(\mathcal{H})$. In particular, a 2-uniform hypergraph is called a simple graph. From now on, by a graph we mean a simple graph. An $r$-uniform hypergraph $\mathcal{H}$ is called $r$-partite if $V(\mathcal{H})$ can be written as a union of $r$ pairwise disjoint subsets (parts) $U_{1}, \ldots, U_{r}$ such that each edge of $\mathcal{H}$ intersects each part $U_{i}$ in one vertex. An $r$-partite hypergraph is called complete if it contains all possible edges. Also, it is said to be balanced if $\left|U_{i}\right|-\left|U_{j}\right| \leq 1$ for each $i, j \in[r]$.

Let $\mathcal{H}$ be a hypergraph and $r$ be an integer, where $r \geq 2$. For pairwise disjoint subsets $U_{1}, \ldots, U_{r} \subseteq V(\mathcal{H})$, the hypergraph $\mathcal{H}\left[U_{1}, \ldots, U_{r}\right]$ is defined to be a subhypergraph of $\mathcal{H}$ whose vertex set is $\cup_{i=1}^{r} U_{i}$ and whose edge set consists of all edges of $\mathcal{H}$ which are contained in $\cup_{i=1}^{r} U_{i}$ and have exactly one element in each $U_{i}$. Note that $\mathcal{H}\left[U_{1}, \ldots, U_{r}\right]$ is an $r$-uniform $r$-partite hypergraph.

A proper coloring of a hypergraph $\mathcal{H}$ is an assignment of colors to the vertices of $\mathcal{H}$ such that there is no monochromatic edge. The chromatic number of a hypergraph $\mathcal{H}$, denoted by $\chi(\mathcal{H})$, is the smallest number $k$ such that there exists a proper coloring of $\mathcal{H}$ with $k$ colors. If there is no such a $k$, we define the chromatic number to be infinite. Let $c$ be a proper coloring of a complete $r$-partite hypergraph $\mathcal{H}$ with parts $U_{1}, \ldots, U_{r}$. The hypergraph $\mathcal{H}$ is colorful (with respect to the coloring $c$ ) whenever for each $i \in[r]$, the vertices in $U_{i}$ receive different colors, that is, $\left|c\left(U_{i}\right)\right|=\left|U_{i}\right|$ for each $i \in[r]$.

Let $\mathcal{H}_{1}=\left(V_{1}, E_{1}\right)$ and $\mathcal{H}_{2}=\left(V_{2}, E_{2}\right)$ be two hypergraphs. For $i=1,2$, the projection $\pi_{i}$ is defined by $\pi_{i}:\left(v_{1}, v_{2}\right) \mapsto v_{i}$. The categorical product of two hypergraphs $\mathcal{H}_{1}$ and $\mathcal{H}_{2}$, defined by Dörfler and Waller in 1980 [10], is the hypergraph $\mathcal{H}_{1} \times \mathcal{H}_{2}$ with vertex set $V_{1} \times V_{2}$ and edge set

$$
\left\{e \subseteq V_{1} \times V_{2}: \pi_{1}(e) \in E_{1}, \pi_{2}(e) \in E_{2}\right\}
$$

In 1992, Zhu [22] proposed the following conjecture as a generalization of Hedetniemi's conjecture.

Conjecture 1.1 ([22]). Let $\mathcal{H}_{1}=\left(V_{1}, E_{1}\right)$ and $\mathcal{H}_{2}=\left(V_{2}, E_{2}\right)$ be two hypergraphs. Then

$$
\chi\left(\mathcal{H}_{1} \times \mathcal{H}_{2}\right)=\min \left\{\chi\left(\mathcal{H}_{1}\right), \chi\left(\mathcal{H}_{2}\right)\right\} .
$$

One can easily derive a proper coloring of $\mathcal{H}_{1} \times \mathcal{H}_{2}$ from a proper coloring of $\mathcal{H}_{1}$ or of $\mathcal{H}_{2}$. Therefore the hard part is to show that $\chi\left(\mathcal{H}_{1} \times \mathcal{H}_{2}\right) \geq \min \left\{\chi\left(\mathcal{H}_{1}\right), \chi\left(\mathcal{H}_{2}\right)\right\}$. Let 
$\mathcal{F}$ be a subhypergraph of $\mathcal{H}_{1} \times \mathcal{H}_{2}$ with the same vertex set and whose edge set consists of minimal edges of $\mathcal{H}_{1} \times \mathcal{H}_{2}$. It is clear that any proper coloring of $\mathcal{F}$ is also a proper coloring of $\mathcal{H}_{1} \times \mathcal{H}_{2}$. This observation shows that Conjecture 1.1 is a generalization of Hedetniemi's conjecture.

For an integer $r$ and a hypergraph $\mathcal{H}$, the $r$-colorability defect of $\mathcal{H}$, denoted by $\operatorname{cd}^{r}(\mathcal{H})$, is the minimum number of vertices that shall be removed from $\mathcal{H}$ so that the hypergraph induced by the remaining vertices admits a proper coloring with $r$ colors.

Let $Z_{r}=\left\{\omega, \omega^{2}, \ldots, \omega^{r}\right\}$ be a multiplicative cyclic group of order $r$ with generator $\omega$. For $X=\left(x_{1}, \ldots, x_{n}\right) \in\left(Z_{r} \cup\{0\}\right)^{n}$, a sequence $x_{i_{1}}, \ldots, x_{i_{m}}$ with $1 \leq i_{1}<\cdots<i_{m} \leq$ $n$ is called an alternating subsequence of $X$ if $x_{i_{j}} \neq 0$ for each $j \in[m]$ and $x_{i_{j}} \neq x_{i_{j+1}}$ for each $j \in[m-1]$. The alternation number of $X$, denoted by $\operatorname{alt}(X)$, is the length of the longest alternating subsequence of $X$. We set $\mathbf{0}=(0, \ldots, 0)$ and $\operatorname{define} \operatorname{alt}(\mathbf{0})=0$. Also, for an $X=\left(x_{1}, \ldots, x_{n}\right) \in\left(Z_{r} \cup\{0\}\right)^{n}$ and for $\varepsilon \in Z_{r}$, define $X^{\varepsilon}=\left\{i: x_{i}=\varepsilon\right\}$. Note that the $r$-tuple $\left(X^{\varepsilon}\right)_{\varepsilon \in Z_{r}}$ uniquely determines $X$ and vice versa. Therefore, with abuse of notations, we can write $X=\left(X^{\varepsilon}\right)_{\varepsilon \in Z_{r}}$. The notation $|X|$ stands for the number of nonzero coordinates of $X$, i.e., $|X|=\sum_{\varepsilon \in Z_{r}}\left|X^{\varepsilon}\right|$. For two vectors $X, Y \in\left(Z_{r} \cup\{0\}\right)^{n}$, we write $X \subseteq Y$ whenever $X^{\varepsilon} \subseteq Y^{\varepsilon}$ for each $\varepsilon \in Z_{r}$.

For a hypergraph $\mathcal{H}$ and a bijection $\sigma:[n] \rightarrow V(\mathcal{H})$, the $r$-alternation number of $\mathcal{H}$ with respect to the permutation $\sigma$ is defined as follows:

$$
\operatorname{alt}_{\sigma}^{r}(\mathcal{H})=\max \left\{\operatorname{alt}(X): E\left(\mathcal{H}\left[\sigma\left(X^{\varepsilon}\right)\right]\right)=\emptyset \text { for all } \varepsilon \in Z_{r}\right\}
$$

The $r$-alternation number of $\mathcal{H}$, denoted by $\operatorname{alt}^{r}(\mathcal{H})$, is equal to $\min _{\sigma}$ alt $_{\sigma}^{r}(\mathcal{H})$ where the minimum is taken over all bijections $\sigma:[n] \rightarrow V(\mathcal{H})$ (for more details see [3]).

For any hypergraph $\mathcal{H}=(V(\mathcal{H}), E(\mathcal{H}))$ and positive integer $r \geq 2$, the general Kneser hypergraph $\mathrm{KG}^{r}(\mathcal{H})$ is an $r$-uniform hypergraph whose vertex set is $E(\mathcal{H})$ and whose edge set is the set of all $r$-subsets of $E(\mathcal{H})$ containing $r$ pairwise disjoint edges of $\mathcal{H}$. Note that by this notation the well-known Kneser hypergraph $\mathrm{KG}^{r}(n, k)$ is the Kneser hypergraph $\mathrm{KG}^{r}\left([n],\left(\begin{array}{c}{[n]} \\ k\end{array}\right)\right)$. For $r=2$, we will rather use $\mathrm{KG}(\mathcal{H})$ than $\mathrm{KG}^{r}(\mathcal{H})$.

Lovász in 1978, by using tools from algebraic topology, proved that $\chi(\mathrm{KG}(n, k))=$ $n-2 k+2$. His paper showed an inspired and deep application of algebraic topology in combinatorics [15]. As a generalization of this result and to confirm a conjecture of Erdős [11], Alon, Frankl, and Lovász [5] proved that the chromatic number of $\mathrm{KG}^{r}(n, k)$ is equal to $\left[\frac{n-(k-1) r}{r-1}\right]$. A different kind of generalization of Lovász's theorem has been obtained by Dol'nikov [9]. He proved that

$$
\chi(\mathrm{KG}(\mathcal{H})) \geq \operatorname{cd}^{2}(\mathcal{H}) .
$$

Then, in 1992, Kříž [13] extended the both latter results by proving that

$$
\chi\left(\mathrm{KG}^{r}(\mathcal{H})\right) \geq\left\lceil\frac{\operatorname{cd}^{r}(\mathcal{H})}{r-1}\right\rceil .
$$

Alishahi and Hajiabolhassan [3] introduced the alternation number as an improvement of colorability defect. Using the $Z_{p}$-Tucker lemma, they proved that

$$
\chi\left(\mathrm{KG}^{r}(\mathcal{H})\right) \geq\left\lceil\frac{|V(\mathcal{H})|-\operatorname{alt}^{r}(\mathcal{H})}{r-1}\right\rceil .
$$


It can be verified that $|V(\mathcal{H})|-\operatorname{alt}^{r}(\mathcal{H}) \geq \operatorname{cd}^{r}(\mathcal{H})$ and the inequality is often strict [3]. Therefore, the preceding lower bound for chromatic number surpasses the Dol'nikov-Kř́žz lower bound. Recently, by an innovative use of the $Z_{p}$-Tucker lemma, Hajiabolhassan and Meunier [12] extended the Alishahi-Hajiabolhassan result (as well as the Dol'nikov-Kř́žz result) to the categorical product of general Kneser hypergraphs as follows.

Theorem A ([12]). Let $\mathcal{H}_{1}, \ldots, \mathcal{H}_{t}$ be hypergraphs and $r$ be an integer, where $r \geq 2$. Then

$$
\chi\left(\operatorname{KG}^{r}\left(\mathcal{H}_{1}\right) \times \cdots \times \mathrm{KG}^{r}\left(\mathcal{H}_{t}\right)\right) \geq\left\lceil\frac{1}{r-1} \min _{i \in[t]}\left(\left|V\left(\mathcal{H}_{i}\right)\right|-\operatorname{alt}^{r}\left(\mathcal{H}_{i}\right)\right)\right\rceil .
$$

Using Theorem A, Hajiabolhassan and Meunier introduced new families of hypergraphs satisfying Zhu's conjecture.

From another point of view, Simonyi and Tardos [20] generalized the Dol'nikov result. Indeed, they proved that for any hypergraph $\mathcal{H}$, if $t=\operatorname{cd}^{2}(\mathcal{H})$, then any proper coloring of $\mathrm{KG}(\mathcal{H})$ contains a complete bipartite subgraph $K_{\lfloor t / 2\rfloor,\lceil t / 2\rceil}$ such that all vertices of this subgraph receive different colors and these different colors occur alternating on the two parts of the bipartite graph with respect to their natural order. Then, this result as well as the Dol'nikov-Kříž result was extended to Kneser hypergraphs by Meunier [19] as the following theorem.

Theorem B ([19]). Let $\mathcal{H}$ be a hypergraph and p be a prime number. Any proper coloring of $\mathrm{KG}^{p}(\mathcal{H})$ contains a colorful, balanced, and complete p-partite subhypergraph $\mathcal{F}$ with $\operatorname{cd}^{p}(\mathcal{H})$ vertices.

It should be mentioned that, in his paper [19], Meunier also generalized Theorem B and proved that this theorem remains true by replacing $\operatorname{cd}^{p}(\mathcal{H})$ with $|V(\mathcal{H})|-\operatorname{alt}^{p}(\mathcal{H})$. In his proof, Meunier used a $Z_{q}$-generalization of a theorem by Ky Fan which is stated in terms of chain maps. Later, by introducing an appropriate generalization of the $Z_{p}$-Tucker lemma, the present second author [2] gave a simple proof for Meunier's result. Moreover, several extensions of Meunier's result can be found in [2]. Another common generalization of the Simonyi-Tardos result and a result by Chen [7, Theorem 7] can be found in [4].

As an improvement of $r$-colorability defect, the equitable $r$-colorability defect was introduced in [1]. For a hypergraph $\mathcal{H}$, the equitable r-colorability defect of $\mathcal{H}$, denoted by $\operatorname{ecd}^{r}(\mathcal{H})$, is the minimum number of vertices that shall be removed so that the subhypergraph induced by the remaining vertices admits a proper equitable $r$-coloring, i.e., a proper $r$-coloring in which the sizes of color classes differ by at most one. Clearly, $\operatorname{ecd}^{r}(\mathcal{H}) \geq$ $\operatorname{cd}^{r}(\mathcal{H})$. As a generalization of Theorem B, it was proved [1] that any proper coloring of $\mathrm{KG}^{p}(\mathcal{H})$ contains a colorful, balanced, and complete $p$-partite subhypergraph $\mathcal{F}$ with $\operatorname{ecd}^{p}(\mathcal{H})$ vertices. It is not difficult to construct a hypergraph $\mathcal{H}$ for which $\operatorname{ecd}^{r}(\mathcal{H})-$ $\operatorname{cd}^{r}(\mathcal{H})$ is arbitrary large. Surpassing the Dol'nikov-Kř̌̌ž lower bound, Abyazi Sani and Alishahi [1] proved

$$
\chi\left(\mathrm{KG}^{r}(\mathcal{H})\right) \geq\left\lceil\frac{\operatorname{ecd}^{r}(\mathcal{H})}{r-1}\right\rceil .
$$

It is worth mentioning that they indeed proved a more general result which in particular implies the prior lower bound. To be more specific, they gave a new lower bound for the chromatic number of a generalization of Kneser hypergraphs introduced by Ziegler which improves substantially Ziegler's lower bound [24, 25]. Furthermore, they compared their 
lower bound with the Dol'nikov-Kř́ž lower bound and the Alishahi-Hajiabolhassan lower bound. In this regard, it was shown that there is a family of hypergraphs $\mathscr{H}$ such that for each hypergraph $\mathcal{H} \in \mathscr{H}$,

$$
\chi\left(\mathrm{KG}^{r}(\mathcal{H})\right)=\left\lceil\frac{\operatorname{ecd}^{r}(\mathcal{H})}{r-1}\right\rceil,
$$

while $\chi\left(\mathrm{KG}^{r}(\mathcal{H})\right)-\left\lceil\frac{\mathrm{cd}^{r}(\mathcal{H})}{r-1}\right\rceil$ and $\chi\left(\mathrm{KG}^{r}(\mathcal{H})\right)-\left\lceil\frac{|V(\mathcal{H})|-\operatorname{alt}^{r}(\mathcal{H})}{r-1}\right\rceil$ are both unbounded for the hypergraphs $\mathcal{H}$ in $\mathscr{H}$. Although there are hypergraphs $\mathcal{H}$ for which $\operatorname{ecd}^{r}(\mathcal{H})-$ $\left(|V(\mathcal{H})|-\operatorname{alt}^{r}(\mathcal{H})\right)$ is arbitrary large, one can construct some hypergraphs $\mathcal{H}$ making $\left(|V(\mathcal{H})|-\operatorname{alt}^{r}(\mathcal{H})\right)-\operatorname{ecd}^{r}(\mathcal{H})$ arbitrary large, see [1].

As the main results of this paper, motivated by the preceding discussion, we simultaneously extend the results by Abyazi Sani and Alishahi [1] and by Hajiabolhassan and Meunier [12] to the following theorems.

Theorem 1.2. Let $\mathcal{H}_{1}, \ldots, \mathcal{H}_{t}$ be hypergraphs. Let $p$ be a prime number and

$$
\eta=\max \left\{\min _{i \in[t]} \operatorname{ecd}^{p}\left(\mathcal{H}_{i}\right), \min _{i \in[t]}\left(\left|V\left(\mathcal{H}_{i}\right)\right|-\operatorname{alt}^{p}\left(\mathcal{H}_{i}\right)\right)\right\} .
$$

Any proper coloring of $\mathrm{KG}^{p}\left(\mathcal{H}_{1}\right) \times \cdots \times \mathrm{KG}^{p}\left(\mathcal{H}_{t}\right)$ contains a colorful, balanced, and complete p-partite subhypergraph $\mathcal{F}$ with $\eta$ vertices.

Remark. The question of whether Theorem 1.2 holds for an arbitrary positive integer $r$ instead of a prime number $p$ is an interesting open question.

Let $c$ be the proper coloring with color set $[C]$. Let $\mathcal{F}$ be the colorful, balanced, and complete $p$-partite subhypergraph whose existence is ensured by Theorem 1.2. Clearly, any color appears in at most $p-1$ vertices of $\mathcal{F}$. Consequently, the previous theorem implies

$$
\chi\left(\mathrm{KG}^{p}\left(\mathcal{H}_{1}\right) \times \cdots \times \mathrm{KG}^{p}\left(\mathcal{H}_{t}\right)\right) \geq\left\lceil\frac{\eta}{p-1}\right\rceil \geq\left\lceil\frac{1}{p-1} \min _{i \in[t]} \operatorname{ecd}^{p}\left(\mathcal{H}_{i}\right)\right\rceil,
$$

which can be extended for an arbitrary $r \geq 2$ as follows.

Theorem 1.3. Let $\mathcal{H}_{1}, \ldots, \mathcal{H}_{t}$ be hypergraphs and $r$ be a positive integer, where $r \geq 2$. Then

$$
\chi\left(\mathrm{KG}^{r}\left(\mathcal{H}_{1}\right) \times \cdots \times \mathrm{KG}^{r}\left(\mathcal{H}_{t}\right)\right) \geq\left\lceil\frac{1}{r-1} \min _{i \in[t]} \operatorname{ecd}^{r}\left(\mathcal{H}_{i}\right)\right\rceil .
$$

Example. In what follows, by introducing some hypergraphs, we compare the two lower bounds presented in Theorems $\mathrm{A}$ and 1.3. Let $n, k, r$ and $a$ be positive integers, where $n \geq r k, n>a$ and $r \geq 2$. Define $\mathcal{H}(n, k, a)$ to be a hypergraph with vertex set $[n]$ and edge set

$$
\{B \subseteq[n]:|B|=k \text { and } B \nsubseteq[a]\} .
$$

Let $\mathrm{KG}^{r}(n, k, a)$ denote the hypergraph $\mathrm{KG}^{r}(\mathcal{H}(n, k, a))$. It was proved [1, Proposition 7] that if either $a \leq 2 k-2$ or $a \geq r k-1$, then $\chi\left(\operatorname{KG}^{r}(n, k, a)\right)=\left\lceil\frac{n-\max \{a, r(k-1)\}}{r-1}\right\rceil$. Indeed, for $a \geq r k-1$, it was proved that

$$
\chi\left(\mathrm{KG}^{r}(\mathcal{H}(n, k, a))\right)=\left\lceil\frac{\operatorname{ecd}^{r}(\mathcal{H}(n, k, a))}{r-1}\right\rceil=\left\lceil\frac{n-a}{r-1}\right\rceil .
$$


One should notice that the chromatic number of $\mathrm{KG}^{r}(\mathcal{H}(n, k, a))$ was left open for several values of $a$ with $2 k-1 \leq a \leq r k-2$. Note that Theorem 1.3 implies the validity of Zhu's conjecture for the family of hypergraphs $\mathrm{KG}^{r}(n, k, a)$ provided that $a \geq r k-1$. What is interesting about the hypergraph $\mathrm{KG}^{r}(\mathcal{H}(n, k, a))$ is the fact that for $r \geq 4$ and $a \geq r k-1$, the value of $\operatorname{ecd}^{r}(\mathcal{H}(n, k, a))-\left(n-\operatorname{alt}^{r}(\mathcal{H}(n, k, a))\right)$ is unbounded. Thus, by the lower bound presented in Theorem A, we cannot derive that the family of hypergraphs $\mathrm{KG}^{r}(n, k, a)$ satisfies Zhu's conjecture. On the other hand, there is a family $\mathscr{H}$ of hypergraphs (see [1]) such that for $\mathcal{H} \in \mathscr{H}$, the value of $\left(n-\operatorname{alt}^{r}(\mathcal{H}(n, k, a))\right)-$ $\operatorname{ecd}^{r}(\mathcal{H}(n, k, a))$ is unbounded. Hence, Theorem A and Theorem 1.3 introduce two somehow complementary lower bounds.

\section{Proofs}

This section is devoted to the proofs of Theorem 1.2 and Theorem 1.3. In the first subsection, we define some necessary tools which will be needed in the rest of the paper. We assume basic knowledge in topological combinatorics. For more details, see [16].

\subsection{Notations and tools}

A simplicial complex is a pair $(V, K)$ where $V$ is a finite nonempty set and $K$ is a family of nonempty subsets of $V$ such that for each $A \in K$, if $\emptyset \neq B \subseteq A$, then $B \in K$. Respectively, the set $V$ and the family $K$ are called vertex set and simplex set of the simplicial complex $(V, K)$. For simplicity of notation and since we can assume that $V=$ $\cup_{A \in K} A$, with no ambiguity, we can point to a simplicial complex $(V, K)$ just by its simplex set $K$. The barycentric subdivision of $K$, denoted by sd $K$, is a simplicial complex whose vertices are the simplices of $K$ and whose simplices are the chains of simplices of $K$ ordered by inclusion.

Let $V$ and $W$ be two sets. We write $V \uplus W$ for the set $V \times\{1\} \cup W \times\{2\}$. Let $K$ and $L$ be two simplicial complexes with vertex sets $V$ and $W$, respectively. We define $K * L$, the join of $K$ and $L$, to be a simplicial complex with vertex set $V \uplus W$ and simplex set $\{A \uplus B: A \in K, B \in L\}$. The join operation is obviously associative: if $K, L, M$ are simplicial complexes, then the simplicial complexes $K *(L * M)$ and $(K * L) * M$ are the same up to a natural relabeling of their vertices. This allows us, if we do not care about the names of the vertices, to use $K * L * M$ for both of $K *(L * M)$ and $(K * L) * M$. The $n$-fold join of $K$, denoted by $K^{* n}$, is a simplicial complex obtained by joining $n$ copies of $K$. By relabeling the vertices of $K^{* n}$, we assume that $K^{* n}$ has vertex set $V(K) \times[n]$ where for each vertex $(v, i) \in V(K) \times[n]$, the index $i$ indicates that the vertex $v$ is considered as a vertex of the $i$ th copy of $K$.

For a prime number $p$, we also consider $Z_{p}$ as a simplicial complex with vertex set $Z_{p}$ and simplex set $\left\{\{\omega\},\left\{\omega^{2}\right\}, \ldots,\left\{\omega^{p}\right\}\right\}$. Clearly $Z_{p}^{* n}$ is a simplicial complex whose vertex set is $Z_{p} \times[n]$ and whose simplices are all nonempty subsets $A \subseteq Z_{p} \times[n]$ such that for each $i \in[n]$, the number of $\varepsilon$ 's for which $(\varepsilon, i) \in A$ is at most one. This observation implies that the simplex set of $Z_{p}^{* n}$ can be identified with the set $\left(Z_{p} \cup\{0\}\right)^{n} \backslash\{\mathbf{0}\}$, i.e., for each simplex $A$ in $Z_{p}^{* n}$, define $A \mapsto\left(x_{1}, \ldots, x_{n}\right)$ where $x_{i}=\varepsilon$ if $(\varepsilon, i) \in A$ and $x_{i}=0$ otherwise. Also, the simplicial complex $\sigma_{p-2}^{p-1}$ is a simplicial complex with vertex set $Z_{p}$ and with simplex set consisting of all nonempty proper subsets of $Z_{p}$. Note that $\left(\sigma_{p-2}^{p-1}\right)^{* n}$ is a simplicial complex with vertex set $Z_{p} \times[n]$ and $\emptyset \neq \tau \subseteq Z_{p} \times[n]$ is a simplex of 
$\left(\sigma_{p-2}^{p-1}\right)^{* n}$ if and only if $\left|\tau \cap\left(Z_{p} \times\{i\}\right)\right| \leq p-1$ for each $i \in[n]$. It is clear that $\left(\sigma_{p-2}^{p-1}\right)^{* n}$ is a free simplicial complex where for each $\varepsilon \in Z_{p}$ and $\left(\varepsilon^{\prime}, i\right) \in Z_{p} \times[n]$, the action is defined by $\varepsilon \cdot\left(\varepsilon^{\prime}, i\right)=\left(\varepsilon \cdot \varepsilon^{\prime}, i\right)$. Let $\tau \in\left(\sigma_{p-2}^{p-1}\right)^{* n}$ be a simplex. For each $\varepsilon \in Z_{p}$, define $\tau^{\varepsilon}=\{(\varepsilon, j):(\varepsilon, j) \in \tau\}$. Also, define

$$
\ell(\tau)=p \cdot h(\tau)+\left|\left\{\varepsilon \in Z_{p}:\left|\tau^{\varepsilon}\right|>h(\tau)\right\}\right|,
$$

where $h(\tau)=\min _{\varepsilon \in Z_{p}}\left|\tau^{\varepsilon}\right|$. As stated above, each $X \in\left(Z_{p} \cup\{0\}\right)^{n} \backslash\{\mathbf{0}\}$ represents a simplex in $Z_{p}^{* n} \subseteq\left(\sigma_{p-2}^{p-1}\right)^{* n}$ and vice versa. Therefore, speaking about $h(X)$ and $\ell(X)$ is meaningful. Indeed, we have

$$
h(X)=\min _{\varepsilon \in Z_{p}}\left|X^{\varepsilon}\right| \quad \text { and } \quad \ell(X)=p \cdot h(X)+\left|\left\{\varepsilon \in Z_{p}:\left|X^{\varepsilon}\right|>h(X)\right\}\right| .
$$

Note that $Z_{p}$ acts freely on $\left(Z_{p} \cup\{0\}\right)^{n} \backslash\{\mathbf{0}\}$ by the action $\varepsilon \cdot X=\left(\varepsilon \cdot x_{1}, \ldots, \varepsilon \cdot x_{n}\right)$, where $\varepsilon \cdot 0$ is defined to be 0 for each $\varepsilon \in Z_{p}$.

Now, we are ready to present the proof of Theorem 1.2. For simplicity, we first assume that $\eta=\min _{i \in[t]} \operatorname{ecd}^{p}\left(\mathcal{H}_{i}\right)$ and then, in Subsection 2.2.2, we sketch the proof for $\eta=$ $\min _{i \in[t]}\left(\left|V\left(\mathcal{H}_{i}\right)\right|-\operatorname{alt}^{p}\left(\mathcal{H}_{i}\right)\right)$. The proof will follow by applying Dold's theorem on a $Z_{p}$-equivariant simplicial map

$$
\begin{aligned}
\lambda: \quad \operatorname{sd}\left(Z_{p}^{* n}\right) & \longrightarrow Z_{p}^{* m} \\
X & \longmapsto(s(X), \nu(X))
\end{aligned}
$$

with $n=\sum_{i=1}^{t}\left|V\left(\mathcal{H}_{i}\right)\right|$ and $m$ as small as possible. Indeed, Dold's theorem implies that if there is such a map $\lambda$, then $m \geq n$. It is worth noting that the idea of using Dold's theorem or some of it specializations such as the $Z_{p}$-Tucker lemma has been used in several articles initiated by a fascinating paper of Matoušek [17]. For instance, see [1, 4, 6, 7, 12, $18,19,24]$. Usually, the most challenging task in using Dold's theorem is how to define the map $\lambda$, especially the sign part $s(X)$. In what follows, we show that some of the techniques used in these works can be fruitfully mixed and extended to get a common generalization. However, some additional tricks are introduced to make these techniques work together. In particular, in our approach, we use a different way to define the sign map $s(X)$ and also we appropriately modify the value function $\nu(X)$. Being more specific, to define the map $\lambda$, we partition $\operatorname{sd}\left(Z_{p}^{* n}\right)=\left(Z_{p} \cup\{0\}\right)^{n} \backslash\{\mathbf{0}\}$ into two subsets $\Sigma_{1}$ and $\Sigma_{2}$, where $\Sigma_{2}$ is the set of vectors $X \in \operatorname{sd}\left(Z_{p}^{* n}\right)$ such that for each $j \in[t]$ and $\varepsilon \in Z_{p}$, the set $\left\{i \in\left[n_{j}\right]: x_{i+\sum_{j^{\prime}=1}^{j-1} n_{j^{\prime}}}=\varepsilon\right\}$ contains some edge of $\mathcal{H}_{j}=\left(\left[n_{j}\right], E_{j}\right)$, and hence $X^{\varepsilon}$ somehow contains a vertex of the hypergraph $\mathrm{KG}^{p}\left(\mathcal{H}_{1}\right) \times \cdots \times \mathrm{KG}^{p}\left(\mathcal{H}_{t}\right)$. For each $X \in \Sigma_{2}$, we define $\nu(X) \in\{\alpha+1, \ldots, m\}$, where $\alpha=n-\eta+p-1$, according to a given proper coloring of $\mathrm{KG}^{p}\left(\mathcal{H}_{1}\right) \times \cdots \times \mathrm{KG}^{p}\left(\mathcal{H}_{t}\right)$ and we define $s(X) \in Z_{p}$ with the help of an auxiliary sign map $s_{3}(-)$. Defining $\lambda(X)$ for the remaining vectors $X$, i.e., $X \in \Sigma_{1}$, is even more difficult and technical which will be done by the use of two auxiliary sign maps $s_{1}(-)$ and $s_{2}(-)$. A larger value of $\eta$ will allow us to make $\alpha$ smaller and consequently $m$ smaller, giving a better bound in the end.

\subsection{Proof of Theorem 1.2}

When $\eta=0$, there is nothing to prove. If $1 \leq \eta \leq p-1$, then consider pairwise disjoint sets $U_{1}, \ldots, U_{p} \subseteq V\left(\mathrm{KG}^{p}\left(\mathcal{H}_{1}\right) \times \cdots \times \mathrm{KG}^{p}\left(\mathcal{H}_{t}\right)\right)$ such that $\left|U_{i}\right|=1$ for $i \leq \eta$ and 
$\left|U_{i}\right|=0$ otherwise. Note that for at least one $i$, we have $U_{i}=\emptyset$. In view of the definitions, the subhypergraph $\mathrm{KG}^{p}\left(\mathcal{H}_{1}\right) \times \cdots \times \mathrm{KG}^{p}\left(\mathcal{H}_{t}\right)\left[U_{1}, \ldots, U_{p}\right]$ which has no edge is clearly balanced and $p$-partite. Furthermore, for any proper coloring of $\mathrm{KG}^{p}\left(\mathcal{H}_{1}\right) \times \cdots \times \mathrm{KG}^{p}\left(\mathcal{H}_{t}\right)$, this subhypergraph is colorful which is desired. Henceforth, we assume that $\eta \geq p$.

For simplicity of notation, assume that $\mathcal{H}_{1}=\left(\left[n_{1}\right], E_{1}\right), \ldots, \mathcal{H}_{t}=\left(\left[n_{t}\right], E_{t}\right)$ and moreover, set $n=\sum_{i=1}^{t} n_{i}$. For each $X=\left(x_{1}, \ldots, x_{n}\right) \in\left(Z_{p} \cup\{0\}\right)^{n} \backslash\{\mathbf{0}\}$, let $X(1) \in\left(Z_{p} \cup\{0\}\right)^{n_{1}}$ be the first $n_{1}$ coordinates of $X, X(2) \in\left(Z_{p} \cup\{0\}\right)^{n_{2}}$ be the next $n_{2}$ coordinates of $X$, and so on, up to $X(t) \in\left(Z_{p} \cup\{0\}\right)^{n_{t}}$ be the last $n_{t}$ coordinates of $X$. Also, for each $j \in[t]$, define $A_{j}(X)$ to be the set of signs $\varepsilon \in Z_{p}$ such that $X(j)^{\varepsilon}$ contains at least one edge of $\mathcal{H}_{j}$. We remind that $X(j)^{\varepsilon}$ is the set of all $i \in\left[n_{j}\right]$ such that $x_{i+\sum_{j^{\prime}=1}^{j-1} n_{j^{\prime}}}=\varepsilon$. Define

$$
\Sigma_{1}=\left\{X \in\left(Z_{p} \cup\{0\}\right)^{n} \backslash\{\mathbf{0}\}: A_{j}(X) \neq Z_{p} \text { for at least one } j \in[t]\right\}
$$

and

$$
\Sigma_{2}=\left\{X \in\left(Z_{p} \cup\{0\}\right)^{n} \backslash\{\mathbf{0}\}: A_{j}(X)=Z_{p} \text { for all } j \in[t]\right\} .
$$

Note that for an $X \in\left(Z_{p} \cup\{0\}\right)^{n} \backslash\{\boldsymbol{0}\}$ and for each $j \in[t]$, if we set $S=\cup_{\varepsilon \in Z_{p}} X(j)^{\varepsilon}$, then $X(j)=\left(X(j)^{\varepsilon}\right)_{\varepsilon \in Z_{p}}$ can be thought of as a partition of vertices of $\mathcal{H}_{j}[S]$ into $p$ color classes, i.e., the vertices in $X(j)^{\varepsilon}$ receive the color $\varepsilon$. Intuitively, the value $h(X(j))$ is then the size of the smallest color class, $\ell(X(j))$ is the maximum possible number of vertices colored by an equitable sub-coloring (not necessarily proper), while $A_{j}(X)$ is the set of colors $\varepsilon \in Z_{p}$ for which there is an $\varepsilon$-monochromatic edge in $\mathcal{H}_{j}[S]$.

\subsubsection{Proof of Theorem 1.2 when $\eta=\min _{i \in[t]} \operatorname{ecd}^{p}\left(\mathcal{H}_{i}\right)$}

In what follows, we first define two sign maps $s_{1}$ and $s_{2}$ playing important roles in the proof. These two maps will help us to define $s(X)$ for each $X \in \Sigma_{1}$.

Definition of $s_{1}(-)$. Let $X \in \Sigma_{1}$ be a vector such that $A_{j}(X) \in\left\{\emptyset, Z_{p}\right\}$ for each $j \in[t]$. Define

$$
B_{j}(X)= \begin{cases}X(j) & \text { if } A_{j}(X)=Z_{p}, \\ \left\{\varepsilon: X(j)^{\varepsilon} \neq \emptyset\right\} & \text { if } A_{j}(X)=\emptyset \text { and } h(X(j))=0, \\ X(j) & \text { if } A_{j}(X)=\emptyset \text { and } h(X(j))>0,\end{cases}
$$

where $\widetilde{X(j)} \in\left(Z_{p} \cup\{0\}\right)^{n_{j}} \backslash\{\mathbf{0}\}$ and for each $\varepsilon \in Z_{p}$, we have

$$
\widetilde{X(j)})^{\varepsilon}= \begin{cases}X(j)^{\varepsilon} & \text { if }\left|X(j)^{\varepsilon}\right|=h(X(j)), \\ \emptyset & \text { otherwise. }\end{cases}
$$

Note that $B_{j}(X)$ may be of two different natures: a vector in $\left(Z_{p} \cup\{0\}\right)^{n_{j}} \backslash\{\boldsymbol{0}\}$ or a proper subset of $Z_{p}$. Now, set $\boldsymbol{B}(X)=\left(B_{1}(X), \ldots, B_{t}(X)\right)$ and

$$
L_{1}=\left\{\boldsymbol{B}(X): X \in \Sigma_{1} \text { and } A_{j}(X) \in\left\{\emptyset, Z_{p}\right\} \text { for all } j \in[t]\right\} .
$$

Note that $L_{1}$ is a subset of

$$
\begin{aligned}
&\left(\left(Z_{p} \cup\{0\}\right)^{n_{1}} \cup\left(2^{Z_{p}} \backslash\left\{Z_{p}\right\}\right)\right) \times \cdots \times \\
&\left(\left(Z_{p} \cup\{0\}\right)^{n_{t}} \cup\left(2^{Z_{p}} \backslash\left\{Z_{p}\right\}\right)\right) \backslash(\{\mathbf{0}, \emptyset\} \times \cdots \times\{\mathbf{0}, \emptyset\}) .
\end{aligned}
$$


For an $\varepsilon \in Z_{p}$ and a vector $\boldsymbol{B}=\left(B_{1}, \ldots, B_{t}\right) \in L_{1}$, we define

$$
\varepsilon \cdot \boldsymbol{B}=\left(\varepsilon \cdot B_{1}, \ldots, \varepsilon \cdot B_{t}\right),
$$

where

$$
\varepsilon \cdot B_{i}= \begin{cases}\left(\varepsilon \cdot x_{1}, \ldots, \varepsilon \cdot x_{n_{i}}\right) & \text { if } B_{i}=\left(x_{1}, \ldots, x_{n_{i}}\right) \in\left(Z_{p} \cup\{0\}\right)^{n_{i}} \backslash\{\mathbf{0}\}, \\ \left\{\varepsilon \cdot z: z \in B_{i}\right\} & \text { if } B_{i} \subsetneq Z_{p} .\end{cases}
$$

With respect to this action, one can simply check that $L_{1}$ is closed and free and furthermore, $\boldsymbol{B}(-)$ is a $Z_{p}$-equivariant map, i.e., $\boldsymbol{B}(\varepsilon \cdot X)=\varepsilon \cdot \boldsymbol{B}(X)$ for each $\varepsilon \in Z_{p}$ and for each $X \in \Sigma_{1}$ such that $A_{j}(X) \in\left\{\emptyset, Z_{p}\right\}$ for each $j \in[t]$. Now, let $s_{1}: L_{1} \rightarrow Z_{p}$ be an arbitrary $Z_{p}$-equivariant map. Note that such a map can be defined by choosing one representative in each orbit and defining the value of the map arbitrarily on this representative.

Definition of $s_{\mathbf{2}}(-)$. Clearly $Z_{p}$ acts freely on

$$
L_{2}=2^{Z_{p}} \times \cdots \times 2^{Z_{p}} \backslash\left(\left\{\emptyset, Z_{p}\right\} \times \cdots \times\left\{\emptyset, Z_{p}\right\}\right)
$$

by the action $\varepsilon \cdot\left(C_{1}, \ldots, C_{t}\right)=\left(\varepsilon \cdot C_{1}, \ldots, \varepsilon \cdot C_{t}\right)$, where $\varepsilon \cdot C_{i}=\left\{\varepsilon \cdot z: z \in C_{i}\right\}$. Similar to the definition of $s_{1}(-)$, let $s_{2}: L_{2} \rightarrow Z_{p}$ be an arbitrary $Z_{p}$-equivariant map.

Set $\alpha=n-\min _{i \in[t]} \operatorname{ecd}^{p}\left(\mathcal{H}_{i}\right)+p-1$. Note that since $\min _{i \in[t]} \operatorname{ecd}^{p}\left(\mathcal{H}_{i}\right) \geq p$, we have $\alpha<n$. For every $j \in[t]$, define the function $\nu_{j}:\left(Z_{p} \cup\{0\}\right)^{n} \backslash\{\mathbf{0}\} \rightarrow \mathbb{N}$ as follows:

$$
\nu_{j}(X)= \begin{cases}|X(j)| & \text { if } A_{j}(X)=Z_{p}, \\ \left|A_{j}(X)\right|+\max \{\ell(Z): Z \subseteq X(j) \text { and } & \text { if } A_{j}(X) \neq Z_{p} . \\ \left.E\left(\mathcal{H}_{j}\left[Z^{\varepsilon}\right]\right)=\emptyset \text { for all } \varepsilon \in Z_{p}\right\} & \end{cases}
$$

We remind the reader that $|X(j)|$ denotes the number of nonzero coordinates in $X(j)$. Now, let $\nu(X)=\sum_{j=1}^{t} \nu_{j}(X)$.

Defining the map $\boldsymbol{\lambda}_{\mathbf{1}}$. Set $\alpha=n-\min _{\varepsilon \in Z_{p}} \operatorname{ecd}^{p}\left(\mathcal{H}_{i}\right)+p-1$. Define the map

$$
\begin{aligned}
\lambda_{1}: \Sigma_{1} & \longrightarrow Z_{p} \times\{1, \ldots, \alpha\} \\
X & \longmapsto(s(X), \nu(X)) .
\end{aligned}
$$

For defining $s(X)$, we consider the following different cases.

- If for each $j \in[t]$, we have $A_{j}(X) \in\left\{\emptyset, Z_{p}\right\}$, then $s(X)=s_{1}(\boldsymbol{B}(X))$.

- If for some $j \in[t]$, we have $A_{j}(X) \notin\left\{\emptyset, Z_{p}\right\}$, then $s(X)=s_{2}\left(A_{1}(X), \ldots, A_{t}(X)\right)$.

Lemma 2.1. The map $\lambda_{1}$ is a $Z_{p}$-equivariant map with no $X, Y \in \Sigma_{1}$ such that $X \subseteq Y$, $\nu(X)=\nu(Y)$ and $s(X) \neq s(Y)$.

Proof. Clearly, $\lambda_{1}$ is a $Z_{p}$-equivariant map since the two maps $s_{1}(-)$ and $s_{2}(-)$ are $Z_{p^{-}}$ equivariant and $\nu(\varepsilon \cdot X)=\nu(X)$ for all $\varepsilon \in Z_{p}$. For a contradiction, suppose that $X$ and $Y$ are two vectors in $\Sigma_{1}$ such that $X \subseteq Y, \nu(X)=\nu(Y)$ and $s(X) \neq s(Y)$. Since $X \subseteq Y$, we have $X(j) \subseteq Y(j)$ and consequently, $A_{j}(X) \subseteq A_{j}(Y)$ for each $j \in[t]$. Additionally, $X(j) \subseteq Y(j)$ implies that

$$
\begin{aligned}
& \left\{\ell(Z): Z \subseteq X(j) \text { and } E\left(\mathcal{H}_{j}\left[Z^{\varepsilon}\right]\right)=\emptyset \quad \forall \varepsilon \in Z_{p}\right\} \subseteq \\
& \left\{\ell(Z): Z \subseteq Y(j) \text { and } E\left(\mathcal{H}_{j}\left[Z^{\varepsilon}\right]\right)=\emptyset \quad \forall \varepsilon \in Z_{p}\right\} .
\end{aligned}
$$


Thus, $\nu_{j}(X) \leq \nu_{j}(Y)$ for each $j \in[t]$. Therefore, the equality $\nu(X)=\nu(Y)$ implies $\nu_{j}(X)=\nu_{j}(Y)$. This equality together with above discussion results in $A_{j}(X)=A_{j}(Y)$ for each $j \in[t]$. This observation leads us to the following cases.

(I) $A_{j}(X) \in\left\{\emptyset, Z_{p}\right\}$ for each $j$. Therefore, $s(X)=s_{1}(\boldsymbol{B}(X))$. Since $A_{j}(X)=$ $A_{j}(Y)$ for each $j$, we have $s(Y)=s_{1}(\boldsymbol{B}(Y))$, Consequently, the fact that $s(X) \neq$ $s(Y)$ implies that $\boldsymbol{B}(X) \neq \boldsymbol{B}(Y)$. Now, let $j_{0}$ be the smallest integer for which $B_{j_{0}}(X) \neq B_{j_{0}}(Y)$. We consider the following different cases.

(1) When $A_{j_{0}}(X)=A_{j_{0}}(Y)=Z_{p}$. In view of the definition of $B_{j_{0}}(-)$, we have $X\left(j_{0}\right) \subsetneq Y\left(j_{0}\right)$. Therefore, the definition of $\nu_{j_{0}}$ implies that $\nu_{j_{0}}(X)<\nu_{j_{0}}(Y)$, which is not possible.

(2) When $A_{j_{0}}(X)=A_{j_{0}}(Y)=\emptyset$. Using $\nu_{j_{0}}(X)=\nu_{j_{0}}(Y)$, we have $\ell\left(X\left(j_{0}\right)\right)=$ $\ell\left(Y\left(j_{0}\right)\right)$. Therefore,

$$
\begin{array}{rl}
p \cdot h\left(X\left(j_{0}\right)\right)+\mid\left\{\varepsilon:\left|X\left(j_{0}\right)^{\varepsilon}\right|\right. & \left.>h\left(X\left(j_{0}\right)\right)\right\} \mid= \\
p & h\left(Y\left(j_{0}\right)\right)+\left|\left\{\varepsilon:\left|Y\left(j_{0}\right)^{\varepsilon}\right|>h\left(Y\left(j_{0}\right)\right)\right\}\right|,
\end{array}
$$

which clearly implies that $h\left(X\left(j_{0}\right)\right)=h\left(Y\left(j_{0}\right)\right)$ and

$$
\left|\left\{\varepsilon:\left|X\left(j_{0}\right)^{\varepsilon}\right|>h\left(X\left(j_{0}\right)\right)\right\}\right|=\left|\left\{\varepsilon:\left|Y\left(j_{0}\right)^{\varepsilon}\right|>h\left(Y\left(j_{0}\right)\right)\right\}\right| .
$$

The fact that $X\left(j_{0}\right) \subseteq Y\left(j_{0}\right)$ results in

$$
\left\{\varepsilon:\left|X\left(j_{0}\right)^{\varepsilon}\right|>h\left(X\left(j_{0}\right)\right)\right\}=\left\{\varepsilon:\left|Y\left(j_{0}\right)^{\varepsilon}\right|>h\left(Y\left(j_{0}\right)\right)\right\} .
$$

Therefore, in view of the definition of $\boldsymbol{B}(-)$, we have $B_{j_{0}}(X)=B_{j_{0}}(Y)$ which is a contradiction.

(II) $A_{j}(X) \notin\left\{\emptyset, Z_{p}\right\}$ for some $j \in[t]$. Since $s(X) \neq s(Y)$, we have

$$
s_{2}\left(A_{1}(X), \ldots, A_{t}(X)\right) \neq s_{2}\left(A_{1}(Y), \ldots, A_{t}(Y)\right) .
$$

Consequently, we must have $\left(A_{1}(X), \ldots, A_{t}(X)\right) \neq\left(A_{1}(Y), \ldots, A_{t}(Y)\right)$. Therefore, there is at least one $j$ for which $A_{j}(X) \neq A_{j}(Y)$ which is not possible.

In what follows, we will define some new notations needed in the rest of proof. Let $c$ be a proper coloring of $\mathrm{KG}^{p}\left(\mathcal{H}_{1}\right) \times \cdots \times \mathrm{KG}^{p}\left(\mathcal{H}_{t}\right)$ with color set $[C]$. For each $X \in \Sigma_{2}$ and each $\varepsilon \in Z_{p}$, define

$$
E^{\varepsilon}(X)=\left\{\left(e_{1}, \ldots, e_{t}\right) \in E_{1} \times \cdots \times E_{t}: e_{j} \subseteq X(j)^{\varepsilon} \text { for each } j \in[t]\right\} .
$$

Note that, in view of the definition of $\Sigma_{2}$, for each $\varepsilon \in Z_{p}$, we have $E^{\varepsilon}(X) \neq \emptyset$. Now, set $\tau_{X}$ to be defined as follows:

$$
\tau_{X}=\left\{(\varepsilon, c(u)): \varepsilon \in Z_{p} \text { and } u=\left(e_{1}, \ldots, e_{t}\right) \in E^{\varepsilon}(X)\right\} .
$$

Note that if we choose $u_{\varepsilon} \in E^{\varepsilon}(X)$ for each $\varepsilon \in Z_{p}$, then $\left\{u_{\varepsilon}: \varepsilon \in Z_{p}\right\}$ is an edge of $\mathrm{KG}^{p}\left(\mathcal{H}_{1}\right) \times \cdots \times \mathrm{KG}^{p}\left(\mathcal{H}_{t}\right)$. Consequently, since $c$ is a proper coloring of $\operatorname{KG}^{p}\left(\mathcal{H}_{1}\right) \times$ $\cdots \times \mathrm{KG}^{p}\left(\mathcal{H}_{t}\right)$, for each $i \in[C]$, there is at least one $\varepsilon \in Z_{p}$ for which $(\varepsilon, i) \notin \tau_{X}$. This observation indicates that $\tau_{X}$ is a simplex of $\left(\sigma_{p-2}^{p-1}\right)^{* C}$. Furthermore, since $E^{\varepsilon}(X) \neq \emptyset$ for each $\varepsilon \in Z_{p}$, we have $\ell\left(\tau_{X}\right) \geq p$. 
Definition of $s_{3}(-)$. For a positive integer $b \in[C]$, let $U_{b}$ be the set consisting of all simplices $\tau \in\left(\sigma_{p-2}^{p-1}\right)^{* C}$ such that $\left|\tau^{\varepsilon}\right| \in\{0, b\}$ for each $\varepsilon \in Z_{p}$. Define $U=\cup_{b=1}^{C} U_{b}$. Choose an arbitrary $Z_{p}$-equivariant map $s_{3}: U \rightarrow Z_{p}$. Also, for each $\tau \in\left(\sigma_{p-2}^{p-1}\right)^{* C}$ with $h=h(\tau)=\min \left|\tau^{\varepsilon}\right|$, define

$$
\widetilde{\tau}=\bigcup_{\varepsilon:\left|\tau^{\varepsilon}\right|=h} \tau^{\varepsilon} .
$$

Note that $\widetilde{\tau}$ is a sub-simplex of $\tau$ which is in $U$. Therefore, $s_{3}(\widetilde{\tau})$ is defined.

Defining the map $\lambda_{2}$. Define the map

$$
\begin{aligned}
\lambda_{2}: \quad \Sigma_{2} & \longrightarrow Z_{p} \times\left\{\alpha+1, \ldots, \alpha-p+1+\max _{X \in \Sigma_{2}} \ell\left(\tau_{X}\right)\right\} \\
X & \longmapsto(s(X), \nu(X)),
\end{aligned}
$$

where $s(X)=s_{3}\left(\widetilde{\tau_{X}}\right)$ and $\nu(X)=\alpha-p+1+\ell\left(\tau_{X}\right)$.

Lemma 2.2. The map $\lambda_{2}$ is a $Z_{p}$-equivariant map with no $X, Y \in \Sigma_{1}$ such that $X \subseteq Y$, $\nu(X)=\nu(Y)$ and $s(X) \neq s(Y)$.

Proof. Obviously, $\lambda_{2}$ is a $Z_{p}$-equivariant map. Suppose for a contradiction that $X$ and $Y$ are two vectors in $\Sigma_{2}$ such that $X \subseteq Y, \nu(X)=\nu(Y)$ and $s(X) \neq s(Y)$. In view of the definition of $\lambda_{2}$, we must have $\ell\left(\tau_{X}\right)=\ell\left(\tau_{Y}\right)$. Using the definition of $\ell(-)$, it implies that $h\left(\tau_{X}\right)=h\left(\tau_{Y}\right)$. From the last equality and $\tau_{X} \subseteq \tau_{Y}$, we deduce that $\widetilde{\tau_{X}}=\widetilde{\tau_{Y}}$ and consequently, $s(X)=s_{3}\left(\widetilde{\tau_{X}}\right)=s_{3}\left(\widetilde{\tau_{Y}}\right)=s(Y)$, which is a contradiction.

In the following lemma, we show that how the existence of an $X$ with large $\ell(X)$ completes the proof.

Lemma 2.3. If there is an $X \in \Sigma_{2}$ with $\ell\left(\tau_{X}\right) \geq q$, then $\mathrm{KG}^{p}\left(\mathcal{H}_{1}\right) \times \cdots \times \mathrm{KG}^{p}\left(\mathcal{H}_{t}\right)$ contains a colorful, balanced, and complete p-partite subhypergraph with q vertices.

Proof. Let $X \in \Sigma_{2}$ be a vector for which we have $\ell\left(\tau_{X}\right) \geq q$. Let $\tau \subseteq \tau_{X}$ be a subsimplex such that $\ell(\tau)=|\tau|=q$. For each $i \in[p]$, set $S_{i}=\left\{j \in[C]:\left(\omega^{i}, j\right) \in \tau\right\}$. First note that $\left\lfloor\frac{q}{p}\right\rfloor \leq\left|S_{i}\right| \leq\left\lceil\frac{q}{p}\right\rceil$ for each $i \in[p]$. Moreover, it is clear that $\sum_{i=1}^{p}\left|S_{i}\right|=$ $q$. For each $i \in[p]$ and $s \in S_{i}$, in view of the definitions of $\tau(X)$ and $S_{i}$, there is a vertex $u_{i, s}=\left(e_{i, 1}^{s}, \ldots, e_{i, t}^{s}\right)$ of $\mathrm{KG}^{p}\left(\mathcal{H}_{1}\right) \times \cdots \times \mathrm{KG}^{p}\left(\mathcal{H}_{t}\right)$ such that $c\left(u_{i, s}\right)=s$ and $e_{i, j}^{s} \subseteq X(j)^{\omega^{i}}$ for each $j \in[t]$. Now, for $i \in[p]$, set $U_{i}=\left\{u_{i, s}: s \in S_{i}\right\}$. Clearly, $\mathrm{KG}^{p}\left(\mathcal{H}_{1}\right) \times \cdots \times \mathrm{KG}^{p}\left(\mathcal{H}_{t}\right)\left[U_{1}, \ldots, U_{p}\right]$ is the desired subhypergraph.

Completing the proof of Theorem 1.2 when $\eta=\min _{i \in[t]} \operatorname{ecd}^{p}\left(\mathcal{H}_{i}\right)$. For completing the proof of Theorem 1.2, we need to use a generalization of the Borsuk-Ulam theorem by Dold, see [8, 16]. Indeed, Dold's theorem implies that if there is a $Z_{p}$-equivariant simplicial map from a simplicial $Z_{p}$-complex $K_{1}$ to a free simplicial $Z_{p}$-complex $K_{2}$, then the dimension of $K_{2}$ should be strictly larger than the connectivity of $K_{1}$.

For simplicity of notation, let

$$
m=\alpha-p+1+\max _{X \in \Sigma_{2}} \ell(\tau(X))
$$


In view of Lemma 2.3, it suffices to show that

$$
\max _{X \in \Sigma_{2}} \ell\left(\tau_{X}\right) \geq \min _{i \in[t]} \operatorname{ecd}^{p}\left(\mathcal{H}_{i}\right)
$$

To this end, define $\lambda:\left(Z_{p} \cup\{0\}\right)^{n} \backslash\{\mathbf{0}\} \rightarrow Z_{p} \times[m]$ such that for each $X \in\left(Z_{p} \cup\right.$ $\{0\})^{n} \backslash\{\boldsymbol{0}\}$, if $X \in \Sigma_{1}$, then $\lambda(X)=\lambda_{1}(X)$, otherwise $\lambda(X)=\lambda_{2}(X)$. In view of Lemma 2.1 and Lemma 2.2, $\lambda(-)$ is a $Z_{p}$-equivariant simplicial map from $\operatorname{sd}\left(Z_{p}^{* n}\right)$ to $Z_{p}^{* m}$. Consequently, according to Dold's theorem, the dimension of $Z_{p}^{* m}$ should be strictly larger than the connectivity of $\operatorname{sd}\left(Z_{p}^{* n}\right)$, that is $m-1>n-2$ as desired.

\subsubsection{Proof of Theorem 1.2 when $\eta=\min _{i \in[t]}\left(\left|V\left(\mathcal{H}_{i}\right)\right|-\operatorname{alt}^{p}\left(\mathcal{H}_{i}\right)\right)$}

In this subsection, we sketch the proof of Theorem 1.2 for the $\eta=\min _{i \in[t]}\left(\left|V\left(\mathcal{H}_{i}\right)\right|-\right.$ alt $\left.^{p}\left(\mathcal{H}_{i}\right)\right)$ case. To this end, we need to slightly modify the proof of Theorem 1.2 in the case of $\eta=\min _{i \in[t]} \operatorname{ecd}^{p}\left(\mathcal{H}_{i}\right)$ as follows.

- Throughout Subsection 2.2.1, replace $\min _{i \in[t]} \operatorname{ecd}^{p}\left(\mathcal{H}_{i}\right)$ by $\min _{i \in[t]}\left(\left|V\left(\mathcal{H}_{i}\right)\right|-\right.$ $\left.\operatorname{alt}^{p}\left(\mathcal{H}_{i}\right)\right)$.

- Use alt $(-)$ instead of function $\ell(-)$ to define each $\nu_{j}(X)$.

- For any $X$ such that $A_{j}(X) \in\left\{\emptyset, Z_{p}\right\}$ for each $j \in[t]$, in the definition of $\lambda_{1}(X)$, set $s(X)$ to be the first nonzero entry of $X$.

With the same approach as in Subsection 2.2.1, it is straightforward to check that Lemmas $2.1,2.2$, and 2.3 are still valid with the preceding modifications. Therefore, again applying Dold's theorem leads us to the desired assertion.

\subsection{Proof of Theorem 1.3}

To prove Theorem 1.3, we reduce this theorem to the prime case of $r$ which is known to be true by the discussion right after Theorem 1.2. One should notice that this reduction is a refinement of the well-known reduction originally due to Kř́ž [14], which has been used in some other papers as well, for instance see [3, 12, 24, 25]. In what follows, we use a similar approach as in [12].

Lemma 2.4. Let $r^{\prime}$ and $r^{\prime \prime}$ be two positive integers. If Theorem 1.3 holds for both $r^{\prime}$ and $r^{\prime \prime}$, then it holds also for $r=r^{\prime} r^{\prime \prime}$.

For two positive integers $s$ and $C$ and a hypergraph $\mathcal{H}$, define a new hypergraph $\mathcal{T}_{\mathcal{H}, C, s}$ as follows:

$$
\begin{aligned}
V\left(\mathcal{T}_{\mathcal{H}, C, s}\right) & =V(\mathcal{H}) \\
E\left(\mathcal{T}_{\mathcal{H}, C, s}\right) & =\left\{A \subseteq V(\mathcal{H}): \operatorname{ecd}^{s}(\mathcal{H}[A])>(s-1) C\right\} .
\end{aligned}
$$

The following lemma can be proved with a similar approach as in [12, Lemma 3].

Lemma 2.5. Let $r$ and $s$ be two positive integers. Then

$$
\operatorname{ecd}^{r s}(\mathcal{H}) \leq r(s-1) C+\operatorname{ecd}^{r}\left(\mathcal{T}_{\mathcal{H}, C, s}\right)
$$

Proof of Lemma 2.4. Using Lemma 2.5 instead of Lemma 3 in the proof of Lemma 1 in [12] leads us to the proof. 


\section{References}

[1] R. Abyazi Sani and M. Alishahi, A new lower bound for the chromatic number of general Kneser hypergraphs, 2017, arXiv:1704.07052v1 [math.CO].

[2] M. Alishahi, Colorful subhypergraphs in uniform hypergraphs, Electron. J. Combin. 24 (2017), \#P1.23, http://www.combinatorics.org/ojs/index.php/eljc/ article/view/v24ilp23.

[3] M. Alishahi and H. Hajiabolhassan, On the chromatic number of general Kneser hypergraphs, J. Comb. Theory Ser. B 115 (2015), 186-209, doi:10.1016/j.jctb.2015.05.010.

[4] M. Alishahi, H. Hajiabolhassan and F. Meunier, Strengthening topological colorful results for graphs, European J. Combin. 64 (2017), 27-44, doi:10.1016/j.ejc.2017.03.011.

[5] N. Alon, P. Frankl and L. Lovász, The chromatic number of Kneser hypergraphs, Trans. Amer. Math. Soc. 298 (1986), 359-370, doi:10.2307/2000624.

[6] G. J. Chang, D. D.-F. Liu and X. Zhu, A short proof for Chen's alternative Kneser coloring lemma, J. Comb. Theory Ser. A 120 (2013), 159-163, doi:10.1016/j.jcta.2012.07.009.

[7] P.-A. Chen, A new coloring theorem of Kneser graphs, J. Comb. Theory Ser. A 118 (2011), 1062-1071, doi:10.1016/j.jcta.2010.08.008.

[8] A. Dold, Simple proofs of some Borsuk-Ulam results, in: H. R. Miller and S. B. Priddy (eds.), Proceedings of the Northwestern Homotopy Theory Conference, American Mathematical Society, Providence, Rhode Island, volume 19 of Contemporary Mathematics, 1983 pp. 65-69, doi:10.1090/conm/019/711043, held at Northwestern University, Evanston, Illinois, March 22 $-26,1982$.

[9] V. L. Dol'nikov, A certain combinatorial inequality, Sib. Math. J. 29 (1988), 375-379, doi: 10.1007/bf00969645.

[10] W. Dörfler and D. A. Waller, A category-theoretical approach to hypergraphs, Arch. Math. 34 (1980), 185-192, doi:10.1007/bf01224952.

[11] P. Erdős, Problems and results in combinatorial analysis, in: Colloquio Internazionale sulle Teorie Combinatorie, Tomo II, Accademia Nazionale dei Lincei, Rome, pp. 3-17, 1976, proceedings of the Convegni Lincei, No. 17, held in Rome, September 3 - 15, 1973.

[12] H. Hajiabolhassan and F. Meunier, Hedetniemi's conjecture for Kneser hypergraphs, J. Comb. Theory Ser. A 143 (2016), 42-55, doi:10.1016/j.jcta.2016.05.002.

[13] I. Kř́ž, Equivariant cohomology and lower bounds for chromatic numbers, Trans. Amer. Math. Soc. 333 (1992), 567-577, doi:10.2307/2154049.

[14] I. Kř́žz, A correction to: "Equivariant cohomology and lower bounds for chromatic numbers" [Trans. Amer. Math. Soc. 333 (1992), 567-577], Trans. Amer. Math. Soc. 352 (2000), 19511952, doi:10.1090/s0002-9947-99-02494-0.

[15] L. Lovász, Kneser's conjecture, chromatic number, and homotopy, J. Comb. Theory Ser. A 25 (1978), 319-324, doi:10.1016/0097-3165(78)90022-5.

[16] J. Matoušek, Using the Borsuk-Ulam Theorem: Lectures on Topological Methods in Combinatorics and Geometry, Universitext, Springer-Verlag, Berlin, 2003, written in cooperation with Anders Björner and Günter M. Ziegler.

[17] J. Matoušek, A combinatorial proof of Kneser's conjecture, Combinatorica 24 (2004), 163 170, doi:10.1007/s00493-004-0011-1.

[18] F. Meunier, The chromatic number of almost stable Kneser hypergraphs, J. Comb. Theory Ser. A 118 (2011), 1820-1828, doi:10.1016/j.jcta.2011.02.010. 
[19] F. Meunier, Colorful subhypergraphs in Kneser hypergraphs, Electron. J. Combin. 21 (2014), \#P1.8, http://www.combinatorics.org/ojs/index.php/eljc/ article/view/v21i1p8.

[20] G. Simonyi and G. Tardos, Colorful subgraphs in Kneser-like graphs, European J. Combin. 28 (2007), 2188-2200, doi:10.1016/j.ejc.2007.04.015.

[21] C. Tardif, Hedetniemi's conjecture, 40 years later, Graph Theory Notes N. Y. 54 (2008), 46-57, http://gtn.kazlow.info/GTN54.pdf.

[22] X. Zhu, On the chromatic number of the products of hypergraphs, Ars Combin. 34 (1992), 25-31.

[23] X. Zhu, A survey on Hedetniemi's conjecture, Taiwanese J. Math. 2 (1998), 1-24, doi:10. 11650/twjm/1500406890.

[24] G. M. Ziegler, Generalized Kneser coloring theorems with combinatorial proofs, Invent. Math. 147 (2002), 671-691, doi:10.1007/s002220100188.

[25] G. M. Ziegler, Erratum: "Generalized Kneser coloring theorems with combinatorial proofs" [Invent. Math. 147 (2002), 671-691], Invent. Math. 163 (2006), 227-228, doi:10.1007/ s00222-005-0466-8. 